Artigo Original

\title{
Efeito das cargas de treinamento sobre parâmetros fisiológicos de futebolistas
}

\author{
Eduardo Zapaterra Campos ${ }^{2}$ \\ Fabio Milioni $^{1}$ \\ Everton Alex Carvalho Zanuto ${ }^{2}$ \\ Pablo Barreto Almeida ${ }^{2}$ \\ Ronaldo Bucken Gobbi ${ }^{1}$ \\ Vitor Luiz de Andrade ${ }^{2}$ \\ Pedro Balikian Junior ${ }^{2}$ \\ Marcelo Papoti ${ }^{3,1}$ \\ ${ }^{1}$ Instituto de Biociências, UNESP - Univ Estadual Paulista, Campus de Rio Claro, Departamento \\ de Educação Física, Rio Claro, SP, Brasil \\ ${ }^{2}$ Faculdade de Ciências e Tecnologia, UNESP - Univ Estadual Paulista, Campus de Presindente \\ Prudente, Departamento de Educação Física, Presidente Prudente, SP,Brasil \\ ${ }^{3}$ Escola de Educação Fisica e Esportes, Universidade de São Paulo - USP \\ de Ribeirão Preto, SP, Brasil
}

\begin{abstract}
Resumo: O objetivo desse estudo foi verificar os efeitos de três semanas de treinamentos com intensidades monitoradas sobre a capacidade aeróbia de futebolistas profissionais. Quatorze futebolistas integrantes de equipe da primeira divisão do Campeonato Brasileiro de 2010, foram avaliados pré e pós três semanas de treinamento. O limiar anaeróbio (LAn) foi determinado pelo método bi-segmentado, para isso quatro esforços submáximos de 800 metros com intensidades de 10, 12, 14 e $16 \mathrm{~km} / \mathrm{h}$ foram aplicados. Trinta três sessões de treinamentos foram quantificadas em zonas de acordo com frequência cardíaca referente ao $L A n\left(F_{L A n}\right): Z 1-10 \%$ abaixo, $Z 2-90-100 \%$ e Z3 - acima da $F_{\text {LAn }}$. Durante os treinamentos os jogadores permaneceram $31,17 \pm 14,86 \%, 42,96 \pm 14,90 \%$ e $25,87 \pm 16,67 \%$ em Z1, Z2 e Z3, respectivamente. Não foram encontradas diferenças significativas no LAn (pré $=13,29 \pm 0,71 \mathrm{~km} \cdot \mathrm{h}^{-1}$; pós $=12,85 \pm 0,90 \mathrm{~km} \cdot \mathrm{h}^{-1}$ ), percepção subjetiva de esforço correspondente ao LAn (pré $=11,53 \pm 1,45$ u.a; pós $=11,23 \pm 1,53$ u.a) e $F_{\text {LAn }}$ (pré $=166,64 \pm 10,69 \mathrm{bpm}$; pós $=174,50 \pm 10,89 \mathrm{bpm}$ ), indicando que três semanas de treinamento são insuficiente para gerar adaptações positivas no LAn de futebolistas.
\end{abstract}

Palavras-chave: Limiar Anaeróbio. Treinamento. Futebol.

\section{Effect of training loads on physiological parameters of soccer players}

Abstract: The aim of this study was to investigate the effects of three weeks of training with intensity monitored on the aerobic capacity of professional soccer players. Fourteen players, members of a first division Brazilian Championship team in 2010, aged $22.78 \pm 3.06$ years were evaluated pre and post three weeks of training. The anaerobic threshold intensity LAn was determined by bi-segmented method, for this four submaximal efforts of 800 meters with intensities 10,12, 14 and $16 \mathrm{~km} / \mathrm{h}$ were applied. Thirty three training sessions were quantified in zones according to heart rate related to the $\mathrm{LAn}\left(\mathrm{FC}_{\mathrm{LAn}}\right): \mathrm{Z1}-10 \%$ below, $\mathrm{Z2}-90-100 \%$ and $\mathrm{Z3}$ - above the $\mathrm{FC}_{\mathrm{LAn}}$. During training participants remained $31.17 \pm 14.86 \%$, $42.96 \%$ and $25.87 \pm 14.90 \pm 16.67 \%$ in $Z 1, Z 2$, and $Z 3$ respectively. There were no significant differences in the LAn (pre $=13,29 \pm 0,71 \mathrm{~km} \cdot \mathrm{h}^{-1}$; post $=12,85 \pm 0,90 \mathrm{~km} \cdot \mathrm{h}^{-1}$ ), perceived exertion (pre = 11,53 $\pm 1,45$ u.a; post $=11,23 \pm 1,53$ u.a) and $\mathrm{FC}_{\mathrm{LAn}}$ (pre $=166,64 \pm 10,69 \mathrm{bpm}$; post $=174,50 \pm 10,89 \mathrm{bpm}$ ) between conditions before and after training, indicating that three weeks of training are insufficient to generate positive changes in soccer players LAn.

Keywords: Anaerobic Threshold. Training. Soccer.

\section{Introdução}

Futebolistas profissionais necessitam de um sistema aeróbio bem desenvolvido para sustentar adequadamente as ações do jogo. Durante uma partida, jogadores de linha percorrem 10-12 km (STOLEN et al., 2005), com frequência cardíaca média de $80-90 \%$ em relação à máxima, e utilizando $70-80 \%$ da capacidade máxima de consumo de oxigênio (CASTAGNA et al., 2011). Em razão dessas condições, o metabolismo aeróbio é severamente mobilizado, sendo responsável por aproximadamente $88 \%$ do total de energia despendida (STOLEN et al. 2005). Ainda, estudos apontam que melhoras significativas do metabolismo aeróbio afetam positivamente o desempenho físico e tático de 
futebolistas (CASTAGNA et al., 2011), bem como aumentam a quantidade de ações técnicas com o envolvimento da bola (HELGERUD et al., 2001), evidenciando, dessa forma, a importância da otimização dessa via metabólica.

Comumente, as variações de desempenho do metabolismo aeróbio durante uma temporada competitiva são verificados de acordo com as alterações do consumo máximo de oxigênio $\left(\mathrm{VO}_{2} \max \right)$ e, principalmente do limiar anaeróbio (LAn). Entretanto, alterações do LAn estão intimamente ligadas com a correta modulação e distribuição das cargas de treinamento empregadas, especialmente durante a pré temporada (CASTAGNA et al., 2011). Para que as cargas impostas sejam de fato eficientes, é necessário 0 monitoramento minucioso das sessões de treinamento, geralmente realizado por meio da frequência cardíaca, percepção subjetiva de esforço e concentração sanguínea de lactato (ENISELER, 2005). Apesar da sua fundamental importância, são escassas as informações na literatura específica a respeito da efetividade das intensidades empregadas durante treinamento periodizado de futebolistas em gerar alterações no LAn, especialmente em situações reais, ou seja, não controladas experimentalmente (STOLEN et al., 2005).

Estudos que verificaram as alterações da capacidade aeróbia em função da distribuição das cargas de treinamento, analisaram períodos de 4 a 8 semanas de pré-temporada (HELGERUD et al., 2001; CASTAGNA et al., 2005; IMPELLIZZERI et al., 2006; HILL-HASS et al., 2009), sem relatos para períodos mais curtos de treinamento ou realizados durante o período competitivo. Além disso, todos esses estudos foram conduzidos com equipes europeias, onde a pré temporada e o calendário competitivo bem definido permitem um período de treinamento adequado, condição distante da realidade brasileira. No Brasil o calendário competitivo é extenso e com poucas folgas, compreende por volta de 42 semanas por temporada, com as equipes disputando de 75 a 85 partidas/ano (GOMES; SOUZA, 2008), o que impossibilita aos clubes a realização de uma prétemporada apropriada. Contudo, nos anos de realização da Copa do Mundo FIFA ${ }^{\circledR}$ de futebol, o Campeonato Brasileiro é interrompido. Essa interrupção possibilitam às equipes melhorarem a condição aeróbia dos jogadores, por meio de uma breve inter-temporada de treinamentos específicos.
Dessa forma o presente estudo teve como principal objetivo verificar o efeito de três semanas de treinamento específico durante a parada do Campeonato Brasileiro para a Copa do Mundo FIFA de 2010 sobre o LAn de jogadores de futebol profissional, além de caracterizar a distribuição das cargas impostas durante essa inter-temporada.

Baseado no estudo de Castagna et al. (2011) que verificaram melhora da condição aeróbia de futebolistas após seis semanas de treinamentos com intensidades monitoradas durante a prétemporada, nossa hipótese é que após uma breve inter-temporada de três semanas de treinamentos específicos, durante a interrupção do Campeonato Brasileiro, seja possível observar melhoras significativas da condição aeróbia de futebolistas profissionais de elite.

\section{Materiais e Métodos}

\section{Amostra}

Participaram do estudo 14 jogadores profissionais de futebol, integrantes de equipe que disputou a $1^{\text {a }}$ divisão do Campeonato Brasileiro de 2010. As características dos sujeitos do presente estudo estão apresentadas na tabela 1 . A porcentagem de gordura foi aferida por meio da técnica de absorptiometria de raios- $X$ de dupla energia (Dual Energy $X$-ray Absorptiometry DEXA - Lunar GE $^{\circledR}$ ) (FREITAS JUNIOR et al., 2012). Os jogadores assinaram um termo de consentimento livre e esclarecido aprovado pelo comitê de Ética da Faculdade de Ciência e Tecnologia da UNESP de Presidente Prudente (processo $n^{\circ} 54 / 2010$ ).

Tabela 1. Caracterização antropométrica dos jogadores avaliados.

\begin{tabular}{lc}
\hline & $\begin{array}{c}\text { Participantes } \\
(\mathbf{n}=\mathbf{1 4})\end{array}$ \\
\hline Idade (anos) & $22,78 \pm 3,06$ \\
Massa Corporal (kg) & $78,37 \pm 7,02$ \\
Gordura Corporal (\%) & $6,95 \pm 2,38$ \\
\hline
\end{tabular}

Procedimentos experimentais

Os sujeitos foram submetidos a duas avaliações progressivas para determinação da velocidade referente ao LAn separadas por três semanas de treinamento. Os protocolos foram realizados em circuito de 200 metros em campo de grama. Para as duas avaliações os sujeitos utilizaram tênis. Os monitoramentos das sessões de treinamento foram realizados por meio da utilização do sistema de posicionamento global 
(GPS Garmin ${ }^{\circledR}$ Forerunner - 405, Suíça). Os pesquisadores não tiveram qualquer influência na elaboração das sessões de treinamento.

\section{Determinação do limiar anaeróbio}

Para determinação da vLAn $(\mathrm{km} / \mathrm{h})$, os atletas foram submetidos à um teste progressivo que consistiu de quatro esforços submáximos (estágios) de 800 metros. As intensidades dos estágios foram, consecutivamente, 10,0, 12,0, 14,0 e $16,0 \mathrm{~km} / \mathrm{h}$, e foram controladas por estímulo sonoro a cada 100 metros. Ao final de cada esforço submáximo, foram coletados $25 \mu \mathrm{L}$ de sangue arterializado do lóbulo da orelha, para determinação da concentração de lactato ([Lac]), frequência cardíaca ( $F C$ ) e percepção subjetiva de esforço (PSE) de acordo com as recomendações de Borg (1982). Os pontos obtidos da relação velocidade versus [Lac] foram submetidos a dois ajustes lineares (método bisegmentado), de modo que o LAn correspondeu ao ponto de intersecção das retas. Através da análise linear entre $F C$ e velocidade foi determinado a FC correspondente ao LAn (FC $L A n)$. A PSE mais próxima do LAn foi definida como a PSE correspondente ao LAn (PSE $E_{L A n}$ ).

\section{Treinamento}

O presente estudo teve caráter descritivo, dessa forma os pesquisadores não tiveram nenhum tipo de interferência ou envolvimento na elaboração das sessões de treinamento.

Os treinamentos foram realizados em dois períodos, manhã e tarde, obedecendo a horários semelhantes de início e término das sessões. As três semanas totalizaram 33 sessões divididas em seis treinos de força, 12 treinos físico-técnicos, oito treinos táticos, dois amistosos e cinco períodos livres.

A quantificação estímulos de treinamento foi realizada por meio da distribuição das intensidades de treinamento em zonas baseadas na $\mathrm{FC}_{\text {LAn }}$ : Z1: $10 \%$ abaixo da $\mathrm{FC}_{\text {LAn }}$, Z2: entre 90$100 \%$ da $\mathrm{FC}_{\text {LAn }}$ e Z3: acima da $\mathrm{FC}_{\text {LAn }}$. Durante todas as sessões de treinamento, assim como no momento das avaliações a FC foi monitorada por um frequencímetro (GPS Garmin ${ }^{\circledR}$ Forerunner 405, Suíça).

\section{Análise sanguínea}

As amostras foram coletadas no lóbulo da orelha em capilares previamente heparinizados. As amostras sanguíneas foram homogeneizadas em eppendorfs contendo $50 \mu \mathrm{L}$ de $\mathrm{NaF}$ a $1 \%$ (fluoreto de sódio) e posteriormente analisadas em lactímetro eletroquímico (Yellow Springs Instruments modelo 1500 Sport, Ohaio, USA).

\section{Tratamento estatístico}

A normalidade dos dados foi analisada pelo teste de Kolmogorov-Smirnov. A comparação das variáveis nas situações pré e pós treinamento foi realizada com a utilização do teste $t$ de student para amostras dependentes com nível de significância fixado em $p<0,05$ e utilizando o software SPSS 17.

\section{Resultados}

Durante as três semanas de treinamento os participantes permaneceram em média 78,8 \pm $37,16 \mathrm{~min} ; 112,66 \pm 64,43 \mathrm{~min}$ e $67,38 \pm 49,06$ min nas zonas Z1, Z2 e Z3, respectivamente. O percentual de permanência em cada zona de intensidade está apresentado na figura 1. O percentual de permanência na Z3 foi estatisticamente diferente de Z2 $(p=0,03)$ (Figura 1).

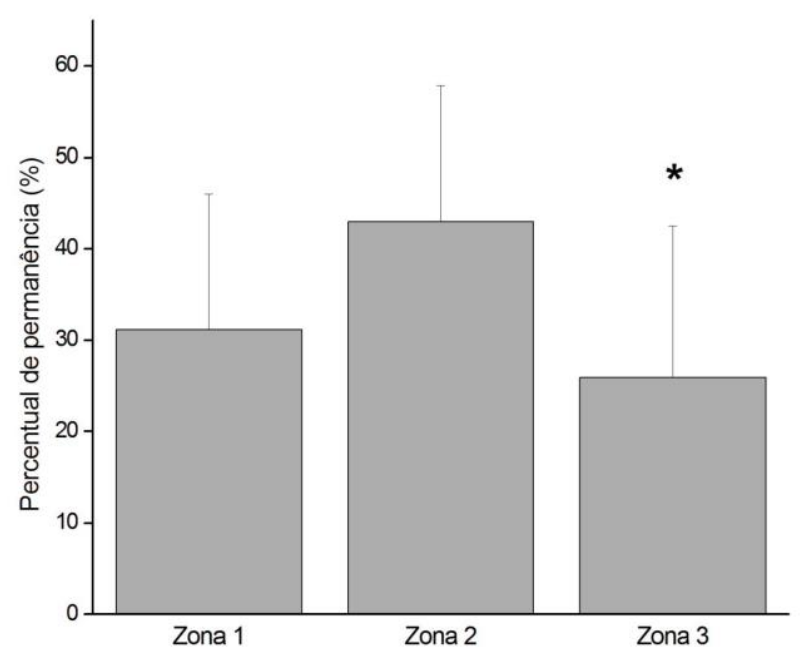

Figura 1. Percentual de permanência (\%) em cada zona de frequência cardíaca. *Diferença significativa de $z 2 p=0,03$.

As [Lac] pré foram significantementes maiores que as [Lac] pós em todos os estágios. A PSE do segundo estágio (E2) na situação pré foi superior à PSE pós (tabela 2). Não foram encontradas diferenças significativas no LAn (pré $=13,29 \pm$ $0,71 \mathrm{~km} \cdot \mathrm{h}^{-1} ;$ pós $=12,85 \pm 0,90 \mathrm{~km} \cdot \mathrm{h}^{-1}, \mathrm{FC}_{\mathrm{LAn}}$ (pré $=166,64 \pm 10,69 \mathrm{bpm}$; pós = 174,50 \pm 10,89 bpm)

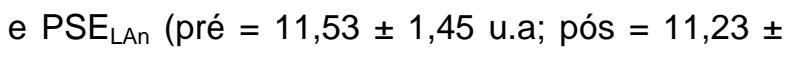
1,53 u.a) entre as condições pré e pós treinamento. 
Tabela 2. Variáveis fisiológicas correspondente a cada estágio dos testes progressivos pré e pós três semanas de treinamento específico.

\begin{tabular}{ccccccc}
\hline $\begin{array}{c}\text { Estágios } \\
\text { (En) }\end{array}$ & $\begin{array}{c}\text { [Lac] } \\
(\mathbf{m m o l} / \mathbf{L})\end{array}$ & $\begin{array}{c}\text { Pré } \\
\text { PSE } \\
(\text { Borg 6-20) }\end{array}$ & $\begin{array}{c}\text { FC } \\
(\mathbf{b p m})\end{array}$ & $\begin{array}{c}\text { [Lac] } \\
(\mathbf{m m o l} / \mathbf{L})\end{array}$ & $\begin{array}{c}\text { Pós } \\
\text { PSE } \\
(\text { Borg 6-20) }\end{array}$ & $\begin{array}{c}\text { FC } \\
(\mathbf{b p m})\end{array}$ \\
\hline E1 & $3,73 \pm 1,18^{*}$ & $8 \pm 1$ & $144 \pm 18$ & $2,96 \pm 0,73$ & $8 \pm 1$ & $148 \pm 10$ \\
E2 & $5,01 \pm 1,56^{*}$ & $10 \pm 2^{*}$ & $161 \pm 16$ & $3,73 \pm 1,05$ & $9 \pm 1$ & $167 \pm 11$ \\
E3 & $6,89 \pm 2,17^{*}$ & $12 \pm 2$ & $179 \pm 10$ & $5,38 \pm 1,30$ & $12 \pm 2$ & $178 \pm 8$ \\
E4 & $9,25 \pm 2,22^{*}$ & $15 \pm 2$ & $180 \pm 19$ & $8,24 \pm 1,79$ & $14 \pm 2$ & $188 \pm 7$ \\
\hline
\end{tabular}

Nota: Média e desvio padrão da concentração de lactato [Lac], frequência cardíaca FC e percepção subjetiva de esforço PSE. *diferença estatística em relação ao pós teste de seu respectivo estágio $(p<0,05)$.

\section{Discussão}

O presente estudo teve como principal objetivo verificar possíveis alterações no LAn de jogadores de futebol profissionais, após três semanas de treinamentos específicos com intensidades monitoradas. Ao contrário da hipótese formulada, três semanas de treinamentos específicos, foram incapazes de gerar adaptações positivas na VLAn de futebolistas profissionais, apesar da diminuição da [Lac] nos esforços submáximos do teste incremental. Além disso, foi verificada a predominância das cargas de treinamento em zonas de baixa e moderada intensidade (Z1 e Z2).

A distribuição de treinamento apresentada durante as três semanas de treinamento difere da encontrada por Castagna et al. (2011). Os autores verificaram predominância (73\%) em Z1 e apenas $8 \%$ em Z3. A distribuição apresentada por Castagna et al. (2011) é semelhante a estudos de atletas de endurance (ESTEVE- LANAO et al. 2005). Contudo, parece que em esportes intermitentes o tempo gasto em zonas de mais alta intensidade determina a magnitude da adaptação aeróbia (CASTAGNA et al. 2011 e IMPELLIZZERI et al. 2005), provavelmente devido ao reduzido volume e duração dos treinamentos.

O valor médio de LAn encontrado no presente estudo é inferior aos achados de outros estudos com jogadores de futebol (CASTAGNA et al. 2011; HELGERUD et al. 2001). Contudo, é importante ressaltar que os jogadores foram avaliados no local em que eram realizados os treinamentos (campo de futebol). Desse modo, a temperatura ambiente característica da região onde os atletas foram avaliados foi mais elevada em comparação às temperaturas utilizadas em avaliações laboratoriais. e recentemente, Barros et al. (2011) verificaram que o calor altera a máxima fase estável de lactato. Os valores da
LAn devem ser analisados nessa perspectiva, pois, em um ambiente controlado, esses valores poderiam diferir dos encontrados no presente estudo.

Não houve diferença estatística no LAn dos atletas investigados no presente estudo, apresentando baixa variação absoluta e percentual desse índice representado pelo $\Delta \mathrm{LAn}$ $\left(-0,44 \pm 1,17 \mathrm{~km} \cdot \mathrm{h}^{-1}\right)$ e percentual de variação ($3,92 \pm 9,74 \%)$, respectivamente. Possivelmente, devido ao "lastro aeróbio" adquirido durante o início da temporada, a melhora do LAn pode ter sido limitada. Porém, a comparação do LAn com o início da temporada não foi realizada.

Outra explicação para a não melhora do LAn pode estar relacionada ao modelo de treinamento utilizado. Eniseler (2005), ao pesquisar a distribuição das intensidades de treinamento de futebolistas durante diferentes tipos de atividades (jogos, jogo modificado, treino técnico e treino tático) em zonas de $\mathrm{FC}$ abaixo de $2 \mathrm{mmol} \cdot \mathrm{L}^{-1} \mathrm{de}$ lactato, entre 2 e $4 \mathrm{mmol} \cdot \mathrm{L}^{-1}$ e acima de $4 \mathrm{mmol} \cdot \mathrm{L}^{-}$ ${ }^{1}$, reportou baixa permanência em zonas de FC acima de $4 \mathrm{mmol} \cdot \mathrm{L}^{-1}$ (alta intensidade) para treinos de caráter tático (apenas 4,5 $\pm 7,9 \%$ do tempo total gasto com esse tipo de atividade). Em concordância aos achados de Eniseler (2005), no presente estudo, das 33 sessões monitoradas, 8 tiveram caráter tático, representando aproximadamente $25 \%$ do total de sessões praticadas que não influenciaram diretamente a melhora da condição aeróbia. Nossos resultados também corroboram com os achados de Castagna et al. (2011) e Impellizzeri et al. (2005). Castagna et al. (2011) encontraram boa associação entre tempo de permanência em zonas de FC acima de $4 \mathrm{mmol} \cdot \mathrm{L}^{-1}$ e melhora na condição aeróbia $\left(r^{2}=0,71\right.$ a 0,42$)$, a medida que Impellizzeri et al. (2005) verificaram correlação 
moderada para a treinamentos de alta intensidade em futebolistas e aumento do consumo máximo de oxigênio em zonas de $\mathrm{FC}$ acima de $4 \mathrm{mmol} / \mathrm{L}$ $(r=0,55, p<0,05)$.

Os jogadores que participaram do presente estudo permaneceram pouco tempo nas zonas acima da $\mathrm{FC}_{\mathrm{LAn}}(25,87 \pm 16,67 \%)$ e a melhora na condição aeróbia de jogadores de futebol parece estar diretamente relacionada ao tempo de permanência em altas intensidades (HELGERUD et al. 2001; IMPELLIZZERI et al. 2006), o que pode ser um contribuinte para a não melhora desse índice. Além disso, Little e Willians (2007) questionam os treinos específicos de futebol, pois os movimentos são esporádicos e difíceis de controlar externamente. Todavia, comprovaram que a FC durante um treino com campo reduzido possuí um baixo coeficiente de variação $(\mathrm{CV} \approx$ $3 \%)$.

As sessões de treino do presente estudo diferem dos relatos de outros estudos que utilizam dimensões, número de jogadores e tempo de estímulo estabelecido (IMPELLIZZERI et al. 2006; LITTLE; WILLIAMS, 2007; DELLAL et al. 2008; SILVA et al. 2011). O coeficiente de variação do tempo de permanência em cada zona do presente estudo foi muito elevado ( $>48 \%$ ) em todas as zonas de intensidade. Essa grande variação na permanência em cada zona de intensidade pode explicar a grande variação na porcentagem de alteração do limiar anaeróbio (média \pm DP: $-3,92 \pm$ $9,74 \%$ ) e a não melhora dos valores de LAn, pois a parte técnica dos jogadores interfere no nível de estresse fisiológico imposto pelo treinamento (DELLAL et al. 2011).

Apesar da não melhora do LAn frente à três semanas de treinamento específico, durante 0 teste foi possível observar redução na concentração de lactato para uma mesma intensidade em todas as velocidades (tabela 2). Possivelmente, o estímulo do treino pode ter sido capaz de aumentar a remoção de lactato em uma mesma intensidade de exercício ou mesmo, reduzir a contribuição anaeróbia para intensidade (DONOVAN e BROOKS, 1983). Além disso, Burgomaster et al. (2006) verificaram redução no metabolismo glicolítico, para uma mesma intensidade, mesmo com pouco tempo de treinamento, em exercícios de alta intensidade e curta duração.

A FC a cada estágio não diferiu entre as situações pré e pós treinamento. A não alteração da $\mathrm{FC}$ dos estágios submáximos e da $\mathrm{FC}_{\mathrm{LAn}}$ colabora com os achados de McMillan et al. (2005) que mostraram que a $\mathrm{FC}_{\mathrm{LAn}}$ não se altera durante uma temporada competitiva. Esse resultado evidencia a utilização dessa variável como um bom parâmetro para prescrição de intensidade de treinamento durante toda temporada.

Duas principais limitações no presente estudo podem ter influenciado a não melhora da LAn. A primeira em relação ao tempo de treinamento entre a avaliação inicial e final, apenas três semanas, a medida que Castagna et al. (2011) e Helgerud et al. (2001) reportaram melhoras em períodos de 6 e 8 semanas, respectivamente. Além disso, esses resultados devem ser visto com cautela, pois, apesar dos atletas almoçarem em horários padronizados (no centro de treinamento), a quantidade, distribuição e alimentação prévia em suas residências, não foi controlados.

A segunda limitação diz respeito à teoria de supercompensação biológica (ISSURIN, 2010), visto que os atletas foram avaliados no dia seguinte ao término dos treinamentos, o que pode ter contribuído para um cenário de supressão das condições fisiológicas, fazendo com que os dados encontrados possam não ter sido fidedignos a real condição aeróbia induzida aos atletas pelas três semanas de treinamento específico, caso as avaliações ocorressem no segundo ou terceiro dia após o término do período de treinamento.

Conclui-se que, três semanas de treinamento não melhoraram estatisticamente o LAn de futebolistas profissionais, apesar da diminuição da [Lac] nos esforços submáximos do teste incremental, o que pode ser considerado uma adaptação aeróbia positiva.

\section{Referências}

BARROS, C. L. M.; MENDES, T. T.; MORTIMER, L. A. C. F.; SIMÕES, H. G.; PRADO, L. S.; WISLOFF, U.; SILAMI-GARCIA, E. Maximal lactate steady state is altered in the heat. International Journal of Sports Medicine, New York, v.32, n.10, p. $749-753,2011$.

BORG, G. A. Psychophysical bases of perceived exertion. Medicine and Science in Sports and Exercise, Philadelphia, v. 14, n. 5, p. 377-381, 1982. 
BURGOMASTER, K. A.; HEIGENHAUSER, G. J. F.; GIBALA, M. J. Effect of short-term sprint interval training on human skeletal muscle carbohydrate metabolism during exercise and time trial performance. Journal of Applied Physiology, Bethesda, v. 100, n. 06, p. 20412047, 2006. Disponível em: $<$ http://iap.physiology.org/content/100/6/2041.full >. Acesso em 12 jul. 2012.

CARLING, C.; BLOOMFIELD, J.; NELSEN, L. The role of motion analysis in elite soccer. Sports Medicine, Auckland, v. 38, n. 10, p. 839-62, 2008.

CASTAGNA, C.; IMPELLIZZERI, F. C.; CHAOUACHI, A.; BORDON, C.; MANZI, V. Effect of training intensity distribution on aerobic fitness variables in elite soccer players: a case study. Journal of Strength and Conditioning Research, Lincoln, v. 25, n. 01, p. 66-71, 2011.

DELLAL, A.; CHAMARI, K.; PINTUS, A.; GIRARD, O.; COTTE, T.; KELLER, D. Heart rate responses during small-sided games and short intermittent running training in elite soccer players: a comparative study. Journal of Strength and Conditioning Research, Lincoln, v. 22, n. 5, p. 1449-57, 2008.

DELLAL, A.; HILL-HAAS, S.; LAGO-PENAS, C.; CHAMARI, K. Small-sided games in soccer: amateur vs. professional players' physiological responses, physical, and technical activities. Journal of Strength and Conditioning Research, Lincoln, v. 25, n. 9, p. 2371-2381, 2011.

DONOVAN, C.; BROOKS, G. Endurance training affects lactate clearance, not lactate production. The American Journal of Physiology, Bethesda, v. 244 n. 7, p. 83-92, 1983. Disponível em: $<$ http://ajpendo.physiology.org/content/244/1/E83.r eprint>, acesso em 26 de mar. 2013.

ENISELER, N. Heart Rate and Blood Lactate concentrations as predictors of physiological load on elite soccer players during various soccer training activities. Journal of Strength and Conditioning Research, Lincoln, v. 19, n. 4, p. 799-804, 2005.

ESTEVE-LANAO, J.; SAN JUAN, A. F.; EARNEST, C. P.; FOSTER, C.; LUCIA, A. How do endurance runners actually train? Relationship with competition performance. Medicine and Science in Sports and Exercise, Philadelphia, v. 37, n. 3, p. 496-504, 2005.

FREITAS JUNIOR, I. F.; MONTEIRO, P. A.; SILVEIRA, L. S.; CAYRES, S. U.; ANTUNES, B. M.; BASTOS, K. N.; CODOGNO, J. S.; SABINO, J. P.; FERNANDES, R. A. Resting heart tate as a predictor of metabolic dysfunctions in obese children and adolescents. BioMed Central

Pediatrics, London, v. 12, n. 5, p. 1-7, 2012.

Disponível em: <

http://www.biomedcentral.com/content/pdf/14712431-12-5.pdf>. Acesso em 9 mai. 2013.

GOBATTO, C. A.; MELLO, M. A.; SIBUYA, C. Y.; AZEVEDO, J. R.; SANTOS, L. A.; KOKUBUN, E. Maximal lactate steady state in rats submitted to swimming exercise. Comparative Biochemistry and Physiology. Part A, Molecular and Integrative Physiology, Victoria, v. 130, n. 01, p. 21-7, 2001 Disponível em: $<$ http://www.sciencedirect.com/science/article/pii/S 1095643301003622>. Acesso em: 12 jul. 2012.

GOMES, A. C.; SOUZA, J. Futebol: treinamento desportivo de alto rendimento. Porto Alegre: Artmed, 2008.

HELGERUD, J.; ENGEN, L. C.; WISLOFF, U.; HOFF, J. Aerobic endurance training improves soccer performance. Medicine and Science in Sports and Exercise, Philadelphia v. 33, n. 11, p. 1925-1931, 2001.

HILL-HAAS, S. V.; DAWSON, B.; IMPELLIZZERI, F. M.; COUTTS, A. J. Physiology of small-sided games training in football: a systematic review. Sports Medicine, Auckland, v. 41, n. 3, p. 199220, 2011.

HOFF, J.; WISLOFF, U.; ENGEN, C. L. Soccer specific aerobic endurance training. British Journal of Sports Medicine, London, v. 36, n. 3, p. 218-222, 2002. Disponível em:

$<$ http://www.ncbi.nlm.nih.gov/pmc/articles/PMC17 24499/?tool=pubmed>. Acesso em: 12 jul. 2012.

IMPELLIZZERI, F. M.; RAMPINNI, E.; MARCORA, S. M. Physiological assessment of aerobic training in soccer. Journal of Sports and Science, Philadelphia, v. 23 n. 6, p. 583-592, 2005.

IMPELLIZZERI, F. M.; MARCORA, S. M.; CASTAGNA, C.; REILLY, T.; SASSI, A.; IAIA, F. M.; RAMPININI, E. Physiological and performance effects of generic versus specific aerobic training in soccer players. International Journal of Sports Medicine, New York, v. 27, n. 6, p. 483492, 2006. doi: http://dx.doi.org/10.1055/s-2005865839 pp.483-92.

ISSURIN, V. B. New horizons for the methodology and physiology of training periodization. Sports Medicine, Auckland, v. 40, n. 3, p. 189-206, 2010. Disponível em:

$<$ http://adisonline.com/sportsmedicine/pages/articl 
eviewer .asp $x$ ?year $=2010$ \&issue $=40030$ \&article $=0$ 0002\&type=abstract>. Acesso em: 12 jul. 2012.

LITTLE, T.; WILLIAMS, A. G. Measures of exercise intensity during soccer training drills with professional soccer players. Journal of Strength and Conditioning Research, Lincoln, v. 21, n. 2, p. 367-71, 2007. Disponível em: $<$ http://ovidsp.tx.ovid.com/sp-

3.5.1a/ovidweb.cgi?T $=J S \& P A G E=$ fulltext\&D=0vft\& $\mathrm{AN}=00124278-200705000-$

00013\&NEWS $=$ N\&CSC $=$ Y \&CHANNEL $=$ PubMed $>$. Acesso em: 12 jul. 2012.

MCMILLAN, K.; HELGERUD, J.; MACDONALD, R.; HOFF, J. Physiological adaptation to soccer specific endurance training in professional youth soccer players. British Journal Sports Medicine, London, v. 39, n.5, p. 273-277, 2005.

RAMPININI, E.; IMPELLIZZERI, F.; CASTAGNA, C.; ABT, G.; CHAMARI, K.; SASSI, A.;

MARCORA, S. M. Factors influencing

physiological responses to small-sided soccer

games. Journal of Sports Science, Philadelphia, v. 25 , n. 6 , p. 659-66, 2007. Disponível em: $<$ http://www.tandfonline.com/doi/abs/10.1080/026 40410600811858?url ver $=$ Z39.882003\&rfr id=ori:rid:crossref.org\&rfr dat=cr pub\% 3dpubmed>. Acesso em: 12 jul. 2012.

REILLY, T. An ergonomics model of the soccer training process. Journal of Sports Science, Philadelphia, v. 23, n. 6, p. 561-72, 2005.

Disponível em:

<http://www.tandfonline.com/doi/abs/10.1080/026 40410400021245?url ver $=$ Z39.88-

2003\&rfr id=ori:rid:crossref.org\&rfr dat=cr pub\% 3dpubmed>. Acesso em: 12 jul. 2012.

SILVA, C. D.; IMPELLIZZERI, F. M.; NATALI, A. J.; LIMA, J. R. P.; BARA-FILHO, M. G.; SILAMIGARCIA, E.; MARINS, J. C. B. Exercise intensity and technical demands of small-sided games in young brazilian soccer players: effects of number of players, maturation, and reliability. Journal of

Strength Conditioning Research, Lincoln, v. 25, n. 10 , p. 2746-51, 2011.

STOLEN, T.; CHAMARI, K.; CASTAGNA, C.; WISLOFF, U. Physiology of soccer: An update. Sports Medicine, Auckland, v. 35, n. 6, p. 50136, 2005.

\section{Endereço:}

Eduardo Zapaterra Campos

Nelson Spacini, 631 Residencial Florenza

Presidente Prudente SP Brasil

19062-110

e-mail: zacampos@yahoo.com.br

Recebido em: 17 de julho de 2012.

Aceito em: 11 de maio de 2013.

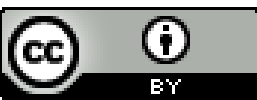

Motriz. Revista de Educação Física. UNESP, Rio Claro, SP, Brasil - elSSN: 1980-6574 - está licenciada sob Creative Commons - Atribuição 3.0 